\title{
COMBINING PROGRAM VISUALIZATION WITH PROGRAMMING WORKSPACE TO ASSIST STUDENTS FOR COMPLETING PROGRAMMING LABORATORY TASK
}

\author{
Elvina Elvina (iD), Oscar Karnalim (iD, Mewati Ayub (iD, Maresha Caroline Wijanto (i) \\ Faculty of Information Technology, Maranatha Christian University (Indonesia) \\ elvina.₹hang@gmail.com,oscar.karnalim@it.maranatha.edu,mewati.ayub@it.maranatha.edu,maresha.cw@it.maranatha.edu
}

Received March 2018

Accepted March 2018

\section{Abstract}

Numerous Program Visualization tools (PVs) have been developed for assisting novice students to understand their source code further. However, none of them are practical to be used in the context of completing programming laboratory task; students are required to keep switching between PV and programming workspace since PV's features are considerably limited for developing programming solution from scratch. This paper combines PV with programming workspace to handle such issue. Resulted tool (which is named PITON) has 13 features extracted from PythonTutor (a program visualization tool), PyCharm (a programming workspace), and student's feedbacks about PythonTutor. According to think-aloud and user study, PITON is more practical to be used than a combination of PythonTutor and PyCharm. Further, its features are considerably helpful; students rated these features as useful and frequently used.

Keywords - Educational tool, Program visualization, Programming workspace, Programming, Computer science education.

\section{Introduction}

When learning programming, one of the most important skills to master is to build a mental model of how source code fragments correspond to runtime processes (Sorva \& Juha, 2013). Learner should know how each instruction works and empirically deduct what will happen if several instructions are executed in sequence. For novice learners (e.g., undergraduate Computer Science students), mastering such skill is not trivial; some of them have no experiences in programming beforehand and most programming concepts are abstract. Therefore, several Program Visualization tools (PVs) have been developed to handle such issue (Sorva, Karavirta \& Malmi, 2013). These tools are expected to train novice learners for building a mental model by visualizing how instructions work on a source code.

Nevertheless, to our knowledge, none of them are specifically designed to assist learners for writing a solution in programming laboratory session; most of them are more focused on visualization, without providing a comprehensive environment to write the code directly. Consequently, when used as a supplementary tool in programming laboratory session, a dedicated programming workspace is still required for writing the code. Learner should keep switching between PV and programming workspace to write the solution and utilize program visualization at once. We would argue that such mechanism is discouraging for learners because, in most occasions, programming tasks should be completed in a limited time.

To fill such gap, this paper proposes a tool which combines PV and programming workspace. This tool is named PITON (Python InTegrated wOrkspace and visualizatioN). It does not only provide a visualization to enhance 
learner's mental model but also a programming workspace environment to develop the code directly. As its name states, PITON is exclusively designed to handle Python source code (Python is chosen as our target language since it is frequently used to introduce programming in most academic institutions (Guo, 2013)). To enhance its effectiveness further, PITON will be developed by considering student feedbacks about PythonTutor, a PV tool which features, to some extent, are similar with PITON. These feedbacks are extracted from (Karnalim \& Ayub, 2017a, 2017b).

\section{Related Work}

According to several studies about CS undergraduate students (Lister, Seppälä, Simon, Thomas, Adams, Fitzgerald et al., 2004; McCracken, Almstrum, Diaz, Guzdial, Hagan, Kolikant et al., 2001), some students find it difficult to learn CS materials. They argue that some CS concepts are abstract and difficult to be understood. Hence, to handle such issue, some educational tools have been developed. Those tools can be classified into two categories: algorithm-oriented and programming-oriented educational tool.

Algorithm-oriented Educational Tool (AT) assists learners to understand well-known algorithms. One of its examples is AP-ASD1 (Christiawan \& Karnalim, 2016). It covers algorithms for manipulating basic data structures (e.g., array, stack, queue, and linked list). This tool is inspired from VisuAlgo (Halim, Koh, Loh \& Halim, 2012), another AT which is focused on visualizing algorithm.

Instead of focusing on algorithms for manipulating data structure, some ATs focus on algorithm strategies. One of them is a work proposed in (Jonathan, Karnalim \& Ayub, 2016). It assists learners to understand brute force, greedy, backtracking, and dynamic programming algorithm from two case studies: 0/1 Knapsack and Minimum Spanning Tree. Another example is a tool proposed in (Zumaytis \& Karnalim, 2017). It covers brute force and branch \& bound algorithm toward Traveling Salesperson Problem.

Considering algorithm strategy is subject to change depends on given problem, some tools assist learners to design their own algorithm strategy. GreedEx (Velázquez-Iturbide \& Pérez-Carrasco, 2009) is an AT which lets learners to try and evaluate their own-defined greedy algorithm. This tool is extended to GreedExCol (Debdi, Paredes-Velasco \& Velázquez-Iturbide, 2015) by incorporating collaborative learning. On the other, Complexitor (Elvina \& Karnalim, 2017) lets learners to understand how algorithm time complexity is defined (by providing the implementation of target algorithm or source code).

It is important to note that not all ATs are focused on well-known algorithms on algorithmic courses. For instance, VPMM (Desai, Kulkarni, Vasant Vaibhav, Varalakshmi \& Mohamed, 2002) focuses on matrix multiplication topic. Another example is a work proposed in (da Silva Lourenço, de Araujo Lima \& Alves de Araújo, 2017) which focuses on network optimization problem.

Programming-oriented Educational Tool (PT) assists learners to understand programming. In most occasions, it is featured with visualization considering illustration may help students to recall explained information (Mayer, 1989). According to the way visualization is used, PT can be further classified into two categories: Visual Programming and Program Visualization tool. Visual Programming tool (VP) lets learners to understand programming without interacting with the source code directly. One of the backgrounds behind such development is the fact that learners struggle with technical details such as remembering syntaxes (Denny, Luxton-Reilly, Tempero \& Hendrickx, 2011). Scratch (Resnick, Silverman, Kafai, Maloney, Monroy-Hernández, Rusk et al., 2009), Alice (Cooper, Dann \& Pausch, 2000) and Greenfoot (Kölling, 2010) are three popular examples of VP. Other two examples of VP are Raptor (Carlisle, Wilson, Humphries \& Hadfield, 2005) and SFC Editor (Watts, 2004). Unique to these tools, interaction is limited to the creation of flowchart.

Nevertheless, since programming cannot be mastered without direct interaction, Program Visualization tool (PV) is introduced. Jeliot 3 (Moreno, Myller, Sutinen \& Ben-Ari, 2004), VILLE (Rajala, Laakso, Kalla \& Salakoski, 2007), and PythonTutor (Guo, 2013) are three examples of such tool. According to their 
long-term evaluation in (Maravić Čisar, Pinter \& Radosav, 2011), (Kaila, Rajala, Laakso \& Salakoski, 2010), and (Karnalim \& Ayub, 2018) respectively, they are considerably helpful to assist novice learners.

Some PVs are equipped with unique features to enhance learners' understanding further. First, SeeC (Egan \& McDonald, 2014) utilizes natural language sentences to provide a clearer understanding. Second, Omnicode (Kang \& Guo, 2017) utilizes live programming environment (i.e., auto-compile environment) on the top of PythonTutor (Guo, 2013) to enhance learners' understanding further. Third, Verificator (Radošević, Orehovački \& Lovrenčić, 2009) limits learners' interaction per compilation to mitigate tremendous errors occurred at once. Last, SRec (Velázquez-Iturbide, Pérez-Carrasco \& Urquiza-Fuentes, 2008 ) incorporates unique view to provide clear visualization for recursion.

Works proposed in (Karnalim \& Ayub, 2017b, 2018) state that PV can be used as a supplementary tool to help undergraduate students in completing their laboratory task. When students are confused about how their code works, they could use PV as an alternative solution to understand their code by themselves. It is true that asking for a help from instructors (i.e., lecturer and their assistants) is more convenient. However, due to the high number of student, instructors are not guaranteed to be able to assist all students.

Our informal observation shows that utilizing PV along with a standard programming workspace develops an issue: the students should copy and paste their code from workspace to PV each time they want to understand how their code works. Such mechanism might be discouraging for students since it takes a considerable amount of time. Further, in most occasions, they have no time to do that due to limited time provided for completing programming task.

One of the solutions to mitigate aforementioned issue is to treat PV as a programming workspace. However, most PVs are not featured with comprehensive workspace's features (e.g., advanced file manipulation). Such condition may discourage students for using given tool. Another solution is to use a tool for Literate Programming (Knuth, 1984), such as Jupyter Notebook (http://jupyter.org/), as a replacement of both PV and programming workspace. Nevertheless, we would argue that given tool is quite complicated to be used and its visualization is not as clear as PV; a tool for Literate Programming is not primarily focused on novice students. Further, its feature for collaboration may lead to illegal activities such as plagiarism.

\section{PITON Design and Implementation}

This paper proposes a tool that combines PV and programming workspace to assist undergraduate students for completing their programming laboratory task. Resulted tool - which is called PITON - is expected to solve application-switching issue that occurs when PV is used along with programming workspace to complete programming task. To gain the benefits from both PV and programming workspace, PITON's features will be derived from existing PV and programming workspace, namely PythonTutor (Guo, 2013) and PyCharm (https://www.jetbrains.com/pycharm/) respectively.

Three major features are derived from PythonTutor: step-by-step visualization, variable content display, and current line highlighting. Step-by-step visualization enables jumping to previous or next execution state to see occurred change. The change for each state is displayed using variables content display and current line highlighting. The former feature displays all involved variables in key-value pairs (where key refers to variable name and value refers to its value) while the latter one marks the current line that is being processed.

Those features are combined with three additional major features derived from PyCharm: standard file manipulation, compile \& run, and syntax highlighting. Standard file manipulation enables creating, opening, updating, and deleting source code file. Compile \& run enables further checking of source code behavior by converting given code to running program. Syntax highlighting enhances students' convenience by displaying source code syntaxes with unique color set (as in typical programming workspace). 
In addition to major features, seven minor features derived from student's feedbacks about PythonTutor (Karnalim \& Ayub, 2017a, 2017b) are also incorporated:

a) No-internet-connection design enables PITON to be used without relying on internet connection. It is implemented by designing PITON as a standalone offline desktop application. This feature is a response of a claim: internet connection in our country is considerably slow.

b) Noticeable input text field accentuates the existence of input text field by providing attractive color and larger size. This feature is a response to a claim: input text field on PythonTutor is considerably hard to be noticed.

c) The multiple-inputs mechanism enables numerous input entities to be given at once. This feature is a response to a claim: providing one input entity at a time is considerably discouraging for executing source code with numerous inputs.

d) Changed variable highlighting marks updated variables with different color during visualization. This feature is a response to a claim: it is difficult to find out which variables are affected by the execution of current instructions.

e) Error message simplification generates simpler error message to avoid confusion. This feature is a response to a claim: standard error messages are not helpful for some students due to their complexity.

f) Once-at-a-time adaptation enables students to adapt with both PV and programming workspace at oce. This feature is a response to a claim: adapting PV takes a considerable amount of time in addition to the time required for adapting programming workspace.

g) Direct visualization enables visualization to be generated directly from programming workspace. This feature is a response to a claim: copying and pasting source code from programming workspace to PV may generate some technical issues.

It is important to note that the rest of feedbacks about PythonTutor (Karnalim \& Ayub, 2017a, 2017b) are excluded from our consideration since, from our perspective, they do not enhance student understanding further. For instance, error-corrector is not provided in PITON even though some students asked for it. We do not want PITON to over-pamper the students, mitigating their programming skill development.

The user interface of PITON can be seen in Figure 1 (for default view) and Figure 2 (for complete view where all panels are shown). It consists of 7 panels: command toolbar, working directory observer, input panel, source code editor, output panel, error panel, and variable content display panel. These panels are referred as A-G respectively in Figure 2.

Command toolbar contains all command buttons for both manipulating and visualizing source code. Student can select one of these buttons to perform an action.

Working directory observer shows all source codes from working directory. Student can select and change current working directory by clicking open directory button on command toolbar. Further, they can also change source code displayed on editor panel by clicking one source code file name on this panel.

Input panel accepts inputs required to execute displayed source code. Three input mechanisms are provided. First, one-input-entity-at-a-time asks student to provide one input entity each time input instruction is being executed. Second, multiple-input-entities-from-user asks student to provide the whole input entities before executing the source code. Third, multiple-input-entities-from-file asks student to provide input in a similar manner as multiple-input-entities-from-user mechanism except that such inputs are provided from a text file.

Source code editor is a panel where student can interact with displayed source code. It is featured with syntax highlighting to enhance source code readability. 
Output panel displays all texts generated from executing displayed source code (both input and output text) in accordance with the execution sequence of input and output instructions. The input text is still displayed on this panel to inform student where the inputs take place.

Error panel displays both syntax and runtime errors which may occur as a result of compiling displayed source code. Unique to this tool, occurred error message is separated from output texts and simplified for easy interpretation. Such simplification is conducted by only displaying the last three lines from the whole error message; since most errors can be solved by only relying on these lines. The example of error message simplification can be seen in Figure 3. It excludes file location and stack trace information.

Variable content display shows any variables declared on executed source code in key-value pair representation. Variables displayed on this panel are limited to primitive variables, strings, array, and matrix. Unique to array and matrix, array is represented as a sequence of blocks while the matrix is represented as a bunch of arrays. An example view of those two can be seen at variable content display panel in Figure 2 as hasil and lst respectively.

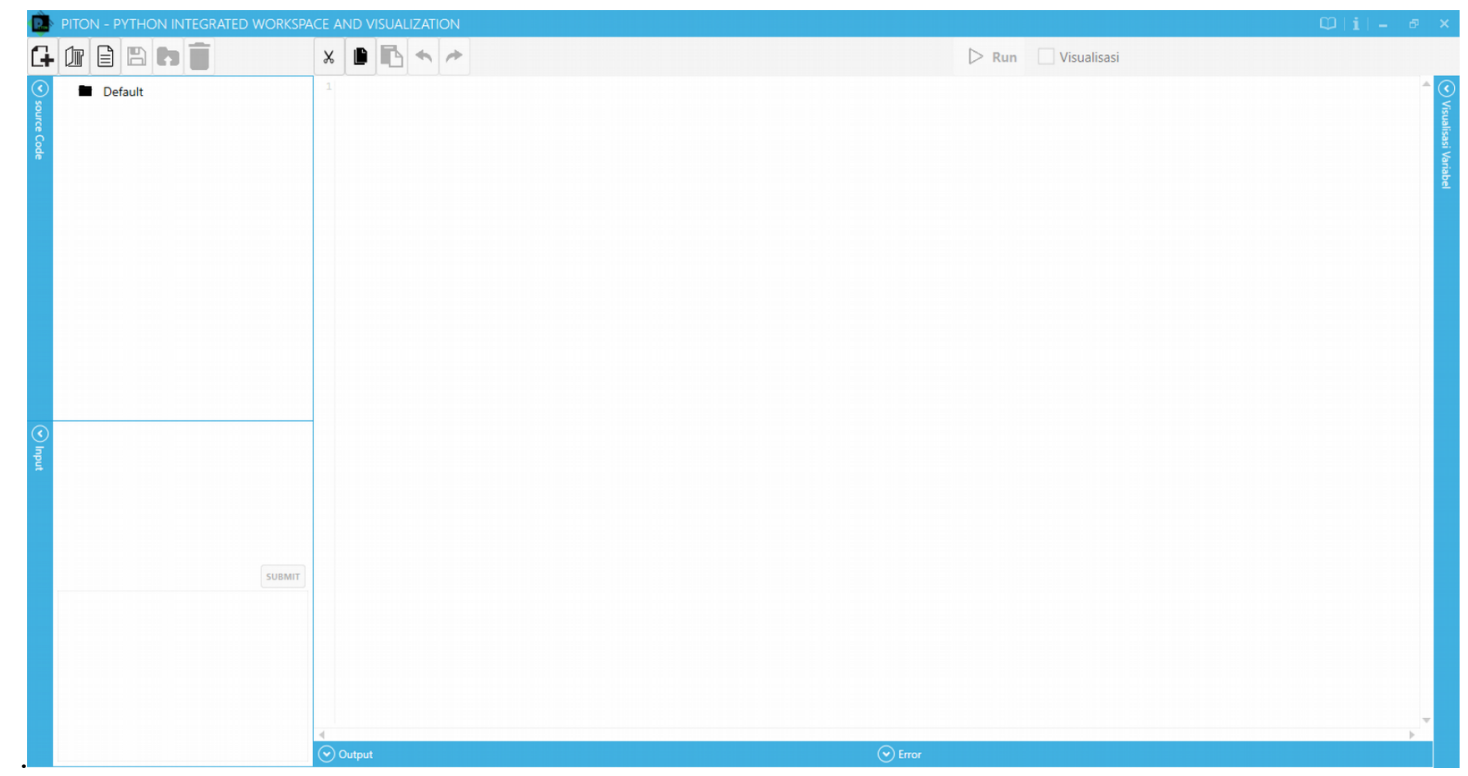

Figure 1. Default user interface of PITON

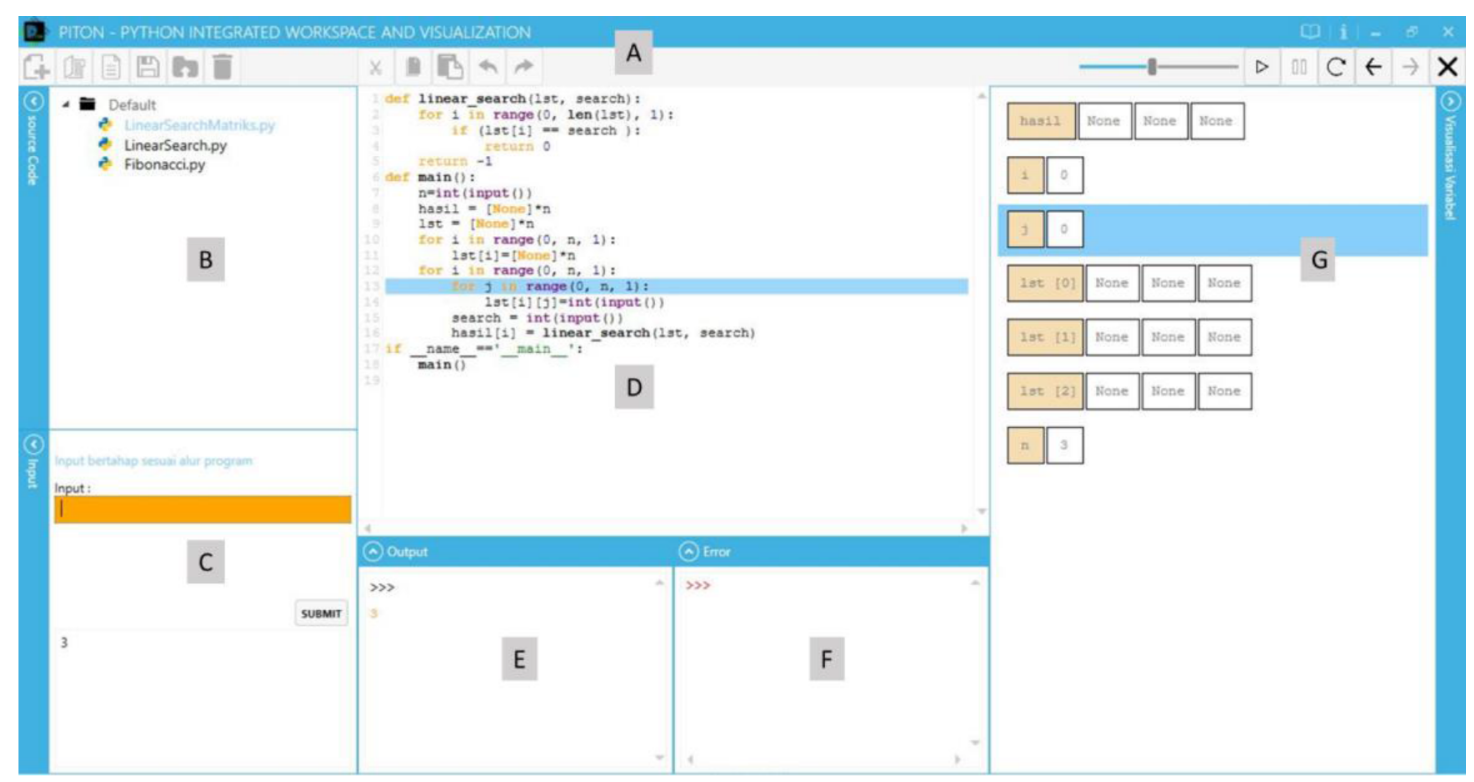

Figure 2. The user interface of PITON when all panels are shown 
Three execution modes are provided in PITON. The first one is standard compile \& run. Student can run his own code, provide the input, and see the result. Figure 4 shows PITON's user interface while running displayed source code. Inputs are given on the bottom-left panel (i.e., input panel) while outputs and error will be displayed at panels right beside input panel (i.e., output and error panel). This mode can be used when program visualization is not required by the student. In contrast, the other two are about executing program visualization (see Figure 2 for PITON's user interface while running displayed source code with program visualization). The only difference between those modes is the way student interacts with visualization. One of them will ask student to press next or previous button during visualization while the other will require no interaction; visualization will be displayed as an automated animation.

Visualization on PITON will be displayed on source code editor, input panel, output panel, and variable content display. Source code editor will highlight currently-executed line (see line 12 at source code editor panel in Figure 2 for an example) where input and output panel will accommodate the interaction between student and source code. For each executed line, the content of all variables will be displayed at variable content display panel; where updated variables will be highlighted with different background color (see $j$ at the variable content display panel in Figure 2 as an example).

For anyone interested to use PITON on their programming course, please kindly inform the first or corresponding author by email. We will send the tool by email.

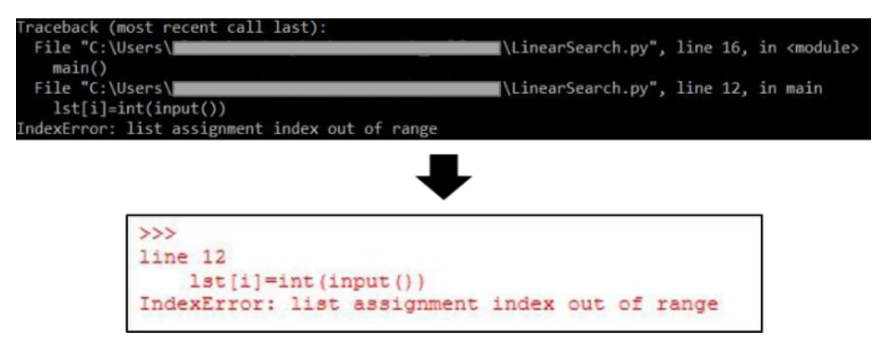

Figure 3. An example of error message simplification

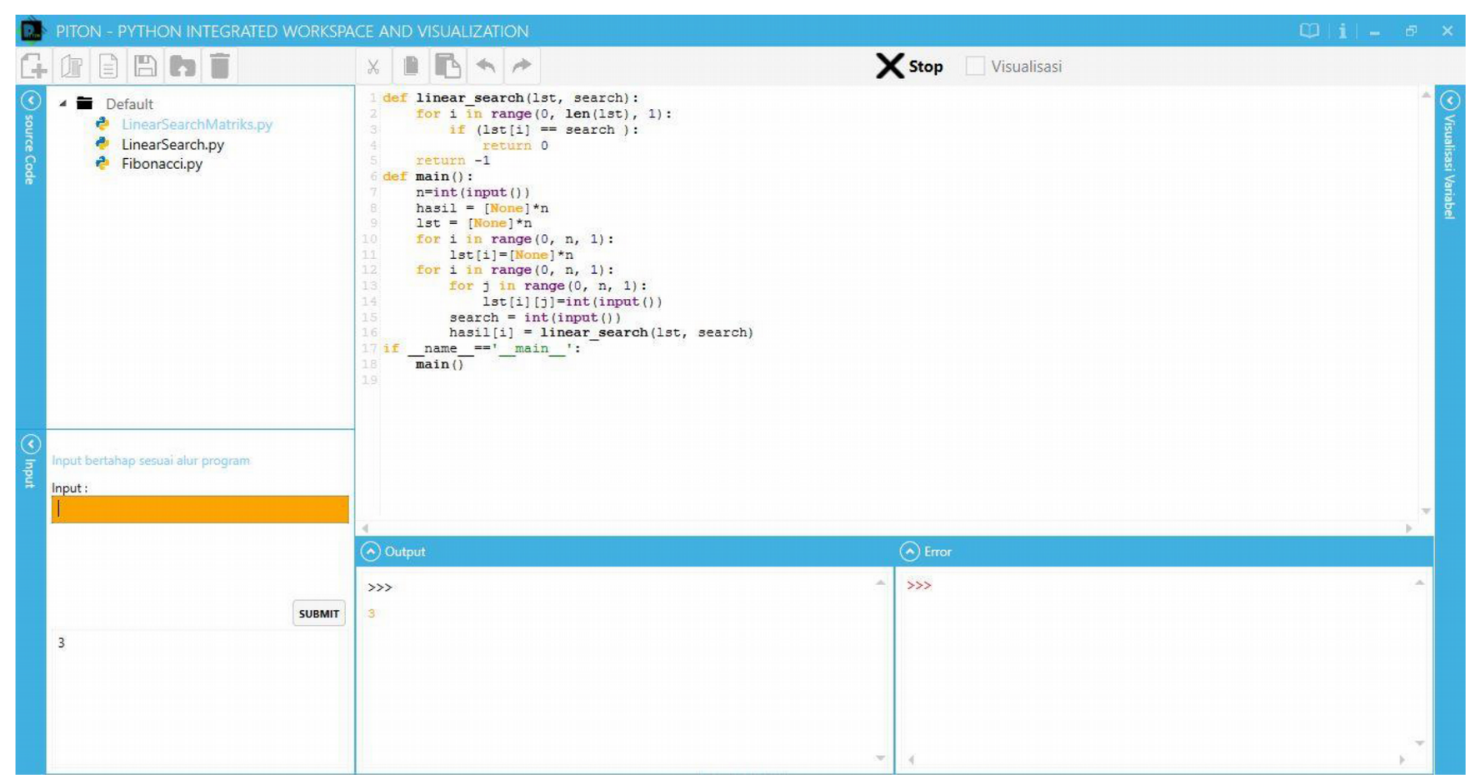

Figure 4. The user interface of PITON while running displayed source code without visualization 


\section{Evaluation}

\subsection{Lecturer-Assistant-Oriented Think-Aloud Study}

We performed a think-aloud study of 8 lecturer assistants from undergraduate CS major; these assistants were expected to know novice students' behavior. Each participant would be asked to solve 4 UVA Online Judge problems (https://uva.onlinejudge.org/) - namely 10050, 11360, 11727, and 12250 - with PITON in 60 minutes (for convenience, each problem had been translated to participant's native language). While solving the problems, these participants were asked to take notes about every thought that might be related to the use of PITON for novice students. These thoughts were then mapped into two categories which are positive and negative feedbacks.

Six positive feedbacks about PITON are collected. First, PITON's features are comprehensive for completing laboratory task. Second, error message provided by PITON is clearer than the standard one (which is displayed by Python compiler). Third, step-by-step visualization is helpful considering each student has his own learning speed. Fourth, multiple-inputs mechanism makes source code testing easier. Fifth, PITON's text editor is convenient to be used due to syntax highlighting. Last, combining programming workspace with a program visualization tool could assist students to complete their laboratory task with ease.

Besides the positive feedbacks, three negative feedbacks are also collected. First, PITON cannot visualize object variables (even the simplest one). Second, some minor bugs occurred (e.g., unexpected notresponding state while visualizing the code). Last, some instructions given on tutorial are quite unclear. Those negative feedbacks (except the first one) were immediately handled upon think-aloud study; the first feedback will be handled on future work.

\subsection{Novice-Student-Oriented User Study}

Evaluation regarding novice students was performed in 9 lecture weeks toward 2 Introductory Programming classes (which consists of 15 and 16 students respectively). Each student would be asked to complete their laboratory task by using either PITON scenario (where only PITON is used), PyCharm scenario (where only PyCharm is used), or PyCharm+PythonTutor scenario (where PyCharm and PythonTutor are used). The scenario of each class per week (including laboratory task material) can be seen in Table 1 . At the first three weeks, both classes were required to complete their task using PyCharm scenario. Later, for each two-weeks, each class tried to use PITON and PyCharm+PythonTutor scenario alternately.

Three evaluation mechanisms were proposed: one quasi-experiment and two questionnaire surveys. Quasiexperiment checks whether PITON scenario is more effective than PyCharm+PythonTutor scenario from students' grade perspective. To do so, for each two-weeks, students' grades for PITON scenario is compared with PyCharm+PythonTutor scenario's using paired t-test; PITON scenario is referred as an experiment group while the other is referred as a control group.

\begin{tabular}{|c|c|c|c|}
\hline Lecture Week & Class A Scenario & Class B Scenario & Material \\
\hline $1^{\text {st }}$ & PyCharm & PyCharm & Introduction \\
\hline $2^{\text {nd }}$ & PyCharm & PyCharm & Nested branching \\
\hline $3^{\text {td }}$ & PyCharm & PyCharm & Loop \\
\hline $4^{\text {th }}$ & PyCharm+PythonTutor & PITON & Nested loop \\
\hline $5^{\text {th }}$ & PITON & PyCharm+PythonTutor & Poid function \\
\hline $6^{\text {th }}$ & PyCharm+PythonTutor & PITON & Function with return value \\
\hline $7^{\text {th }}$ & PITON & PyCharm+PythonTutor & Array with function \\
\hline $8^{\text {th }}$ & PITON & PyCharm+PythonTutor & PITON \\
\hline $9^{\text {th }}$ & PyCharm+PythonTutor & Pron & Arth \\
\hline
\end{tabular}

Table 1. Scenario distribution per class 
In contrast, two questionnaire surveys check PITON's effectiveness from students' perspectives. The first survey compares PITON with PyCharm and PythonTutor. Each student is required to rate 7 statements in 7-point Likert scale (where 1 refers to completely disagree, 4 refers to neutral, and 7 refers to completely agree). This survey was conducted at the end of $5^{\text {th }}$ week (where students had tried all scenarios). Whereas, the second one measures how useful PITON's features and how frequent they are used while completing laboratory task. Each student is required to rate PITON's features regarding those two aspects in 10-points scale. For usefulness metric, 0 refers to not useful and 10 refers to extremely useful; whereas, for frequency metric, 0 refers to never used and 10 refers to always used. This survey was conducted at the end of $9^{\text {th }}$ week. It is important to note that those two surveys were taken at two different times to avoid human fatigue.

Prior to conducting quasi-experiment, students' grade means from the $4^{\text {th }}$ to $9^{\text {th }}$ week should be calculated. Figure 5 shows that two findings can be deducted regarding grade means. First, class A has lower chance to get affected by PITON than class B; its grade mean is more consistent across lecture weeks. Second, students in our evaluation could generate higher score when a closely-related topic is given. The $5^{\text {th }}$ week (nested loop) and the $7^{\text {th }}$ week (a function with return value) generates higher mean than their predecessor week (which has closely-related material). It is important to note that the $9^{\text {th }}$ week (array with function) does not share similar behavior as the $5^{\text {th }}$ and $7^{\text {th }}$ week since its material combines two topics (i.e., array and function) instead of deepening its predecessor's material.

Table 2 shows that only function material on class B generates significance through t-test ( $\mathrm{p}$-value $<0.01$ ). However, such significance favors the combination of PyCharm and PythonTutor instead of PITON; grade mean for PyCharm+PythonTutor scenario is higher than PITON's. Such un-patterned result is due to the fact that most students did not use PythonTutor during PyCharm+PythonTutor scenario; for students, switching between PyCharm and PythonTutor during laboratory session is not practical. This phenomenon implicitly changes a comparison between PyCharm+PythonTutor and PITON scenario to a comparison between PyCharm and PITON scenario. When compared to PyCharm, PITON was slightly less convenient to complete programming task considering students had used PyCharm longer. It is important to note that this rationale also backs up two findings regarding students' grade mean: class A generated considerably consistent grade mean and both classes generated higher grade mean when related topic is given regardless of used scenario.

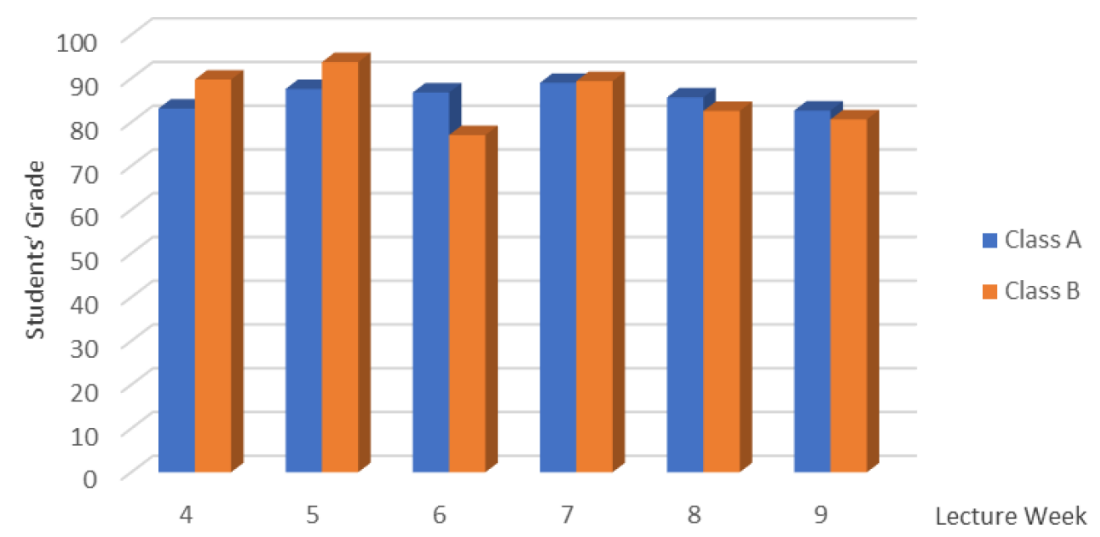

Figure 5. Students' grade means per class for quasi-experiment

\begin{tabular}{|c|c|c|c|}
\hline \multirow{2}{*}{ Material } & \multirow{2}{*}{ Involved Weeks } & Class A & P-Value \\
\cline { 3 - 4 } & Loop & $4^{\text {th }}$ and $5^{\text {th }}$ week & 0.474 \\
\hline Function & $6^{\text {th }}$ and $7^{\text {th }}$ week & 0.312 & 0.188 \\
\hline Array & $8^{\text {th }}$ and $9^{\text {th }}$ week & 0.389 & 0.0045 \\
\hline
\end{tabular}

Table 2. P-value for each two-weeks in quasi-experiment 
In addition to un-patterned result, an implicit finding can also be deducted from quasi-experiment: students tend not to use program visualization tool when it is separated from programming workspace. In other words, PITON is beneficial to be used for completing laboratory task; it combines program visualization tool with programming workspace.

The survey which compares PITON with PyCharm and PythonTutor consists of 7 statements; five of them compares PITON with PythonTutor while the other two compares PITON with PyCharm. The detail of survey statements and their resulted rate mean can be seen in Table 3. In general, students agree that PITON outperforms PythonTutor and PyCharm in aspects mentioned in survey statements; all resulted rate means are higher than upper quartile (that is close to completely agree). Among given statements, $\mathrm{C} 01$ generates the highest rate mean since internet connection in our country is limited. On the other, the lowest rate mean is generated by $\mathrm{C} 07$ (which is about error message simplification); students implicitly do similar simplification as proposed in our feature when they read an error message displayed in PyCharm.

The survey which measures how useful PITON's features and how frequent they are used while completing laboratory task consists of 13 questions where each question refers to one PITON's feature. The first six questions are related to major features while the others are related to minor features (see Table 4 for mapping details).

\begin{tabular}{|c|l|c|}
\hline ID & \multicolumn{1}{|c|}{ Statement } & Rate mean \\
\hline C01 & $\begin{array}{l}\text { PITON is more convenient to be used than PythonTutor since no internet connection is } \\
\text { required }\end{array}$ & 6.543 \\
\hline C02 & $\begin{array}{l}\text { Changed variable highlighting (which cannot be found on PythonTutor) is helpful to } \\
\text { understand how given source code works }\end{array}$ & 6.257 \\
\hline C03 & $\begin{array}{l}\text { Current line highlighting on PITON is clearer than current line highlighting on PythonTutor } \\
\text { (since it highlights the whole line) }\end{array}$ & 6.029 \\
\hline C04 & $\begin{array}{l}\text { Standard file manipulation in PITON is helpful to complete laboratory task (this feature } \\
\text { cannot be found on PythonTutor) }\end{array}$ & 6.4 \\
\hline C05 & $\begin{array}{l}\text { PITON's option to compile \& run without visualization is helpful to complete laboratory } \\
\text { task (this feature cannot be found on PythonTutor) }\end{array}$ & 6.286 \\
\hline C06 & $\begin{array}{l}\text { Step-by-step visualization (which cannot be found on PyCharm) is helpful to understand } \\
\text { how given source code works }\end{array}$ & 6 \\
\hline C07 & $\begin{array}{l}\text { Error message simplification (which cannot be found on PyCharm) generates more } \\
\text { understandable error message }\end{array}$ & 5.571 \\
\hline
\end{tabular}

Table 3 . The $1^{\text {st }}$ survey: statements and their rate mean

\begin{tabular}{|c|l|c|}
\hline ID & \multicolumn{1}{|c|}{ Statement } & Feature Type \\
\hline F01 & Step-by-step visualization & Major \\
\hline F02 & Variable content display & Major \\
\hline F03 & Current line highlighting & Major \\
\hline F04 & Standard file manipulation & Major \\
\hline F05 & Compile \& run & Major \\
\hline F06 & Syntax highlighting & Major \\
\hline F07 & No-internet-connection design & Minor \\
\hline F08 & Noticeable input textfield & Minor \\
\hline F09 & Multiple-input mechanism & Minor \\
\hline F10 & Changed variable highlighting & Minor \\
\hline F11 & Error message simplification & Minor \\
\hline F12 & Once-at-a-time adaptation & Minor \\
\hline F13 & Direct visualization & Minor \\
\hline
\end{tabular}

Table 4 . The $2^{\text {nd }}$ survey: involved features 
When perceived from usefulness metric, Figure 6 shows that all features are considerably useful; their usefulness degree mean is higher than 6 (i.e., a minimum threshold in positive degree category). F13 generates the highest usefulness degree; it generates 9.071 of 10 . We would argue that such finding is natural considering students tend not to use program visualization tool when it is separated from programming workspace (as stated in quasi-experiment result). In contrast, F12 generates the lowest usefulness degree. It is the only usefulness degree that is lower than upper quartile. Further observation shows that F12's feature (i.e., once-at-a-time adaptation) was not felt useful since students had adapted other program visualization tool (i.e., PythonTutor) and programming workspace (i.e., PyCharm) beforehand.

From frequency perspective, Figure 6 shows that all features are used in more than $50 \%$ occasions; their frequency degree mean is higher than 5. Implicit features (e.g., once-at-a-time adaptation) do not generate the highest degree since students did not feel that they use it that frequent (even though they do so). We would argue that the finding is natural; the impact of some implicit features is not extreme enough to be felt in all occasions.

Among given statements, F07 is rated the highest (i.e., 8.286 of 10). Students felt that no-internetconnection design (which is F07's feature) is frequently used despite its implicit behavior. This finding supports our previous finding from the $1^{\text {st }}$ survey: the need of internet connection plays an important role when designing tool for areas with limited internet connection. The lowest frequency degree is resulted by F02; most students did not concentrate on variable content display (which is F02's feature) during visualization. They concentrated more on current line highlighting (i.e., F03's feature) and changed variable highlighting (i.e., F10's feature).

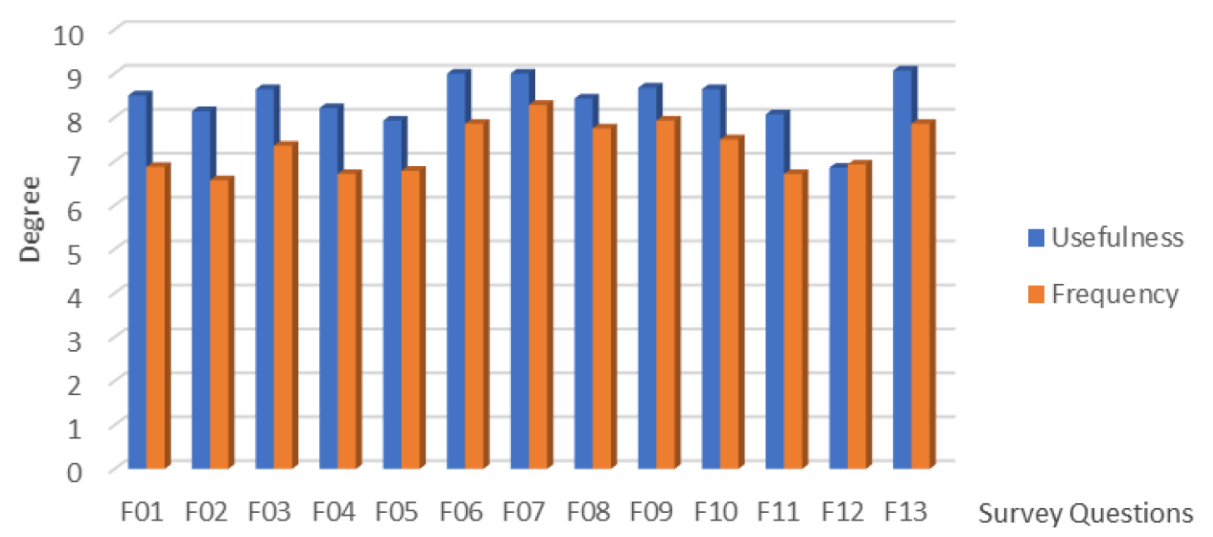

Figure 6. Usefulness and frequency result toward PITON's features

\section{Conclusions and Future Works}

This paper presents a combination of program visualization tool and programming workspace, namely PITON. Using this tool, novice students could utilize program visualization while completing their laboratory task with ease. PITON is featured with 3 major features from PythonTutor (i.e., program visualization tool), 3 major features from PyCharm (i.e., programming workspace), and 7 minor features extracted from student's feedbacks about PythonTutor (Karnalim \& Ayub, 2017a, 2017b). According to our evaluation, PITON is preferred than the combination of PythonTutor and PyCharm for completing laboratory task (even though it is still less convenient when compared to PyCharm as a programming workspace). Further, all features proposed in PITON are helpful since they are perceived as useful and frequently used by students.

For future work, we plan to observe PyCharm's convenient features and apply them to PITON. Further, in response to negative feedback from think-aloud study, we also plan to extend PITON's scope to cover 
object visualization (especially data-structure-related objects such as Stack, Queue, and Linked List). This feature is expected to help students for learning advanced topic.

\section{Declaration of Conflicting Interests}

The authors declared no potential conflicts of interest with respect to the research, authorship, and/or publication of this article.

\section{Funding}

This work is financially supported by a grant from Maranatha Christian University, Indonesia.

\section{References}

Carlisle, M.C., Wilson, T.A., Humphries, J.W., \& Hadfield, S.M. (2005). RAPTOR: a visual environment for teaching algorithmic problem solving. Acm Sigcse Bulletin, 37(1), 176-180. Retrieved from: https://dl.acm.org/citation.cfm?id=1047411

Christiawan, L., \& Karnalim, O. (2016). AP-ASD1: An Indonesian Desktop-based Educational Tool for Basic Data Structure Course. Jurnal Teknik Informatika Dan Sistem Informasi (JuTISI), 2(1). Retrieved from http://jutisi.maranatha.edu/index.php/jutisi/article/view/422

Cooper, S., Dann, W., \& Pausch, R. (2000). Alice: a 3-D tool for introductory programming concepts. In Journal of Computing Sciences in Colleges, 15, 107-116. Retrieved from https://dl.acm.org/citation.cfm? $\underline{\mathrm{id}=364161}$

da Silva Lourenço, W., de Araujo Lima, S.J., \& Alves de Araújo, S. (2017). TASNOP: A tool for teaching algorithms to solve network optimization problems. Computer Applications in Engineering Education. https://doi.org/10.1002/cae.21864

Debdi, O., Paredes-Velasco, M., \& Velázquez-Iturbide, J.Á. (2015). GreedExCol, A CSCL tool for experimenting with greedy algorithms. Computer Applications in Engineering Education, 23(5), 790-804. Retrieved from: https://onlinelibrary.wiley.com/doi/full/10.1002/cae.21655

Denny, P., Luxton-Reilly, A., Tempero, E., \& Hendrickx, J. (2011). Understanding the syntax barrier for novices. In Proceedings of the 16th annual joint conference on Innovation and technology in computer science education ITiCSE '11, 208. New York, New York, USA: ACM Press. https://doi.org/10.1145/1999747.1999807

Desai, S., Kulkarni, S., Vasant Vaibhav, Varalakshmi, M., \& Mohamed, P. (2002). VPMM: visualization of parallel matrix multiplication algorithms. Journal of Computing Sciences in Colleges, 33(1), 24-31. Retrieved from: https://dl.acm.org/citation.cfm?id=3144610

Egan, M.H., \& McDonald, C. (2014). Program visualization and explanation for novice C programmers. In Proceedings of the Sixteenth Australasian Computing Education Conference - Volume 148. Australian Computer Society, Inc. Retrieved from: https://dl.acm.org/citation.cfm?id=2667496

Elvina, E., \& Karnalim, O. (2017). Complexitor: An Educational Tool for Learning Algorithm Time Complexity in Practical Manner. ComTech: Computer, Mathematics and Engineering Applications, 8(1), 21. https://doi.org/10.21512/comtech.v8i1.3783

Guo, P.J. (2013). Online python tutor: embeddable web-based program visualization for cs education. In Proceeding of the 44th ACM technical symposium on Computer science education - SIGCSE '13, 579. New York, New York, USA: ACM Press. https://doi.org/10.1145/2445196.2445368

Halim, S., Koh, Z. C., Loh, V.B.H., \& Halim, F. (2012). Learning Algorithms with Unified and Interactive Web-Based Visualization. Olympiads in Informatics, 6, 53-68. Retrieved from: https://www.mii.lt/olympiads in informatics/pdf/INFOL099.pdf 
Jonathan, F.C., Karnalim, O., \& Ayub, M. (2016). Extending The Effectiveness of Algorithm Visualization with Performance Comparison through Evaluation-integrated Development. In Seminar Nasional Aplikasi Teknologi Informasi (SNATI). Retrieved from:

http://www.jurnal.uii.ac.id/index.php/Snati/article/view/6263

Kaila, E., Rajala, T., Laakso, M.J., \& Salakoski, T. (2010). Effects of Course-Long Use of a Program Visualization Tool. In Australasian Computing Education Conference. Brisbane. Retrieved from: https://dl.acm.org/citation.cfm?id=1862234

Kang, H., \& Guo, P.J. (2017). Omnicode: A Novice-Oriented Live Programming Environment with Always-On Run-Time Value Visualizations. In The 30th ACM Symposium on User Interface Software and Technology (UIST. Retrieved from: https://dl.acm.org/citation.cfm?id=3126632

Karnalim, O., \& Ayub, M. (2017a). The Effectiveness of a Program Visualization Tool on Introductory Programming: A Case Study with PythonTutor. CommIT (Communication and Information Technology) Journal, 11(2). Retrieved from: http://journal.binus.ac.id/index.php/commit/article/view/3704

Karnalim, O., \& Ayub, M. (2017b). The Use of PythonTutor on Programming Laboratory Session: Student Perspectives. KINETIK, 2(4). Retrieved from:

http://kinetik.umm.ac.id/index.php/kinetik/article/view/442

Karnalim, O., \& Ayub, M. (2018). A Quasi-Experimental Design to Evaluate the Use of PythonTutor on Programming Laboratory Session. International Journal of Online Engineering (iJOE), 14(2), 155-164. Retrieved from: http://online-journals.org/index.php/i-joe/article/view/8067

Knuth, D.E. (1984). Literate Programming. The Computer Journal, 27(2). Retrieved from: https://academic.oup.com/cominl/article/27/2/97/343244

Kölling, M. (2010). The greenfoot programming environment. ACM Transactions on Computing Education (TOCE), 10(4), 14. Retrieved from: https://dl.acm.org/citation.cfm?id=1868361

Lister, R., Seppälä, O., Simon, B., Thomas, L., Adams, E. S., Fitzgerald, S., et al. (2004). A multi-national study of reading and tracing skills in novice programmers. In Working group reports from ITiCSE on Innovation and technology in computer science education - ITiCSE-WGR '04, 36, 119. New York, New York, USA: ACM Press. https://doi.org/10.1145/1044550.1041673

Maravić Čisar, S., Pinter, R., \& Radosav, D. (2011). Effectiveness of Program Visualization in Learning Java: a Case Study with Jeliot 3. International Journal of Computers Communications \& Control, 6(4), 668. https://doi.org/10.15837/ijccc.2011.4.2094

Mayer, R.E. (1989). Systematic Thinking Fostered by Illustration in Scientific Text. Journal of Educational Psychology, 81(2). Retrieved from:

http://psycnet.apa.org/record/1989-34646-001 https://doi.org/10.1037/0022-0663.81.2.240

McCracken, M., Almstrum, V., Diaz, D., Guzdial, M., Hagan, D., Kolikant, Y., et al. (2001). A MultiNational, Multi-Institutional Study of Assessment of Programming Skill of First-year CS Students. ACM SIGSCE Bulletin, 33(4). https:// doi.org/10.1145/572133.572137

Moreno, A., Myller, N., Sutinen, E., \& Ben-Ari, M. (2004). Visualizing programs with Jeliot 3. In Proceedings of the working conference on Advanced visual interfaces - AVI '04, 373. New York, New York, USA: ACM Press. https://doi.org/10.1145/989863.989928

Radošević, D., Orehovački, T., \& Lovrenčić, A. (2009). Verificator: Educational Tool for Learning Programming. Informatics in Education, 8(2). Retrieved from: http://citeseerx.ist.psu.edu/viewdoc/summary? $\underline{\text { doi }=10.1 \cdot 1 \cdot 148.9441}$ 
Rajala, T., Laakso, M.-J., Kalla, E., \& Salakoski, T. (2007). VILLE: a language-independent program visualization tool. In Proceedings of the Seventh Baltic Sea Conference on Computing Education Research. 88, 151159. Darlinghurst: Australian Computer Society. Retrieved from: https://dl.acm.org/citation.cfm? $\underline{\mathrm{id}=2449340}$

Resnick, M., Silverman, B., Kafai, Y., Maloney, J., Monroy-Hernández, A., Rusk, N., et al. (2009). Scratch: Programming for All. Communications of the ACM, 52(11), 60. https://doi.org/10.1145/1592761.1592779

Sorva, J., \& Juha. (2013). Notional machines and introductory programming education. ACM Transactions on Computing Education, 13(2), 1-31. https://doi.org/10.1145/2483710.2483713

Sorva, J., Karavirta, V., \& Malmi, L. (2013). A Review of Generic Program Visualization Systems for Introductory Programming Education. ACM Transactions on Computing Education, 13(4), 1-64. https://doi.org/10.1145/2490822

Velázquez-Iturbide, J.Á., \& Pérez-Carrasco, A. (2009). Active learning of greedy algorithms by means of interactive experimentation. In Proceedings of the 14th annual ACM SIGCSE conference on Innovation and technology in computer science education - ITiCSE '09 (Vol. 41, p. 119). New York, New York, USA: ACM Press. https://doi.org/10.1145/1562877.1562917

Velázquez-Iturbide, J.Á., Pérez-Carrasco, A., \& Urquiza-Fuentes, J. (2008). SRec: : an animation system of recursion for algorithm courses. In Proceedings of the 13th annual conference on Innovation and technology in computer science education - ITiCSE '08, 40, 225. New York, New York, USA: ACM Press. https://doi.org/10.1145/1384271.1384332

Watts, T. (2004). The SFC editor a graphical tool for algorithm development. Journal of Computing Sciences in Colleges, 20(2), 73-85. Retrieved from: https://dl.acm.org/citation.cfm?id=1040159

Zumaytis, S., \& Karnalim, O. (2017). Introducing an Educational Tool for Learning Branch \& Bound Strategy. Journal of Information Systems Engineering and Business Intelligence, 3(1), 8.

https://doi.org/10.20473/jisebi.3.1.8-15

Published by OmniaScience (www.omniascience.com)

Journal of Technology and Science Education, 2018 (www.jotse.org)

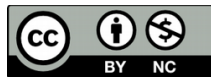

Article's contents are provided on an Attribution-Non Commercial 4.0 Creative commons International License. Readers are allowed to copy, distribute and communicate article's contents, provided the author's and JOTSE journal's names are included. It must not be used for commercial purposes. To see the complete licence contents, please visit https://creativecommons.org/licenses/by-nc/4.0/. 